\title{
Value of automatic DNA image cytometry for diagnosing lung cancer
}

\author{
ANQI SHI, WANG MIN, LAI XIANG, WU XU and TAO JIANG \\ Department of Respiratory and Critical Care Medicine, \\ The First Affiliated Hospital of Chongqing Medical University, Chongqing 400016, P.R. China
}

Received June 18, 2017; Accepted February 2, 2018

DOI: $10.3892 / \mathrm{ol} .2018 .8723$

\begin{abstract}
The present study aimed to investigate the diagnostic value of automatic DNA image cytometry (DNA-ICM) for diagnosing lung cancer. A total of three different types of samples from 465 cases were included: Bronchoalveolar lavage fluid (BALF), 386 samples; pleural effusion cases, 70 samples; and fine-needle aspiration procedures, 9 samples. Two methods, liquid-based cytology (LBC) and automatic DNA-ICM, were used to assess the samples, and the pathological results of 120/465 cases were reviewed. The results of DNA-ICM were compared with those of LBC and pathology. There were 57 cases of lung cancer without aneuploidy and 49 cases without evidence of malignant tumor, but with the presence of heteroploid cells. The positive diagnostic rate for BALF samples using LBC was significantly higher compared with that for DNA-ICM $(\mathrm{P}<0.05)$. No statistically significant difference was observed in the positive diagnostic rate between DNA-ICM and LBC in pleural effusion samples. For DNA-ICM in BALF, pleural effusion and all samples, no statistically significant differences were identified between the positive diagnostic rates of lung squamous carcinoma and lung adenocarcinoma. The positive diagnostic rate of LBC combined with DNA-ICM was not significantly improved. In non-small cell lung cancer (NSCLC) cases, the difference in the maximum value of DNA (DNAmax) was positively correlated with tumor stage $(\mathrm{P}<0.05)$, but no significant correlations were observed among DNA max, tumor type and tumor location. In small-cell lung cancer (SCLC) cases, no significant correlations were observed among DNAmax, tumor staging or tumor location. The differences in the DNAmax values of squamous cell carcinoma, adenocarcinoma, SCLC and NSCLC were not statistically significant. In the present study, the area under
\end{abstract}

Correspondence to: Professor Tao Jiang, Department of Respiratory and Critical Care Medicine, The First Affiliated Hospital of Chongqing Medical University, 1 Youyi Road, Chongqing 400016, P.R. China

E-mail: 562775743@qq.com

Key words: lung cancer, diagnosis, DNA image cytometry, liquid-based cytology, bronchoalveolar lavage fluid, pleural effusion the receiver operating characteristic curve for LBC (0.936) was significantly greater compared with that for DNA-ICM (0.766) $(\mathrm{P}<0.05)$. DNA-ICM has medium diagnostic value in lung cancer, and the DNAmax was positively correlated with tumor stage in NSCLC. DNA-ICM may serve as a supplement to $\mathrm{LBC}$, but it is not recommended as a sole procedure for lung cancer screening.

\section{Introduction}

Lung cancer is one of the most common types of malignant carcinoma worldwide and is associated with gradually increasing rates of morbidity and mortality $(1,2)$. Therefore, it is important to establish a timely and accurate method for diagnosing lung cancer in the clinic. Liquid-based cytology (LBC) was listed as one of the differential diagnostic standards for lung cancer by the World Health Organisation in 2004 (3). LBC have many advantages, such as a well preserved cellular structure, a reduction in the number of inadequate smears and an even distribution of cells (4). Based on these advantages compared with traditional cytological examination, LBC has been widely used in the diagnosis of lung cancer (5-7). However, as the results of LBC are primarily dependent on the judgement of pathologists, who examine samples with their naked eyes, the possibility of false-negative results may not be excluded. DNA image cytometry (DNA-ICM) has gained attention for its advantages, including objectivity, convenience and a high positive rate, in diagnosing various malignant cancer types. Several studies have explicitly demonstrated the significance of DNA-ICM in the diagnosis of cervical, oesophageal, gastric and oral mucosal cancer (8-14). The present study aimed to investigate the diagnostic value of DNA-ICM for lung cancer based on a comparison with LBC and pathological biopsy (Fig. 1).

\section{Materials and methods}

Common materials. The present study was approved by the Ethics Committee of the First Affiliated Hospital of Chongqing Medical University (Chongqing, China). Written informed consent was obtained from all patients. A total of 600 samples were collected from the Respiratory Department of the First Affiliated Hospital of Chongqing Medical University between August 2015 and November 2015. Among them, 
135 samples were excluded from the experimental group due to unsatisfactory results following automatic DNA-ICM, including 120 bronchoalveolar lavage fluid (BALF) samples, 12 pleural effusion samples and 3 fine-needle aspiration (FNA) samples. As a result, a total of 465 samples were included in the experimental group, including 386 BALF samples, 70 samples from cases of pleural effusion and 9 samples from FNA procedures. A total of $315(67.7 \%)$ samples were from male patients and $150(32.3 \%)$ were from female patients, with an age range of $16-83$ years (mean age, $58.50 \pm 13.11$ years). Two methods, LBC and automatic DNA-ICM, were used to evaluate these samples. During the next six months (between August 2015 and May 2016), the pathological results of 120 samples from bronchoscopy, endobronchial ultrasound-guided transbronchial needle aspiration and surgical procedures were also reviewed,

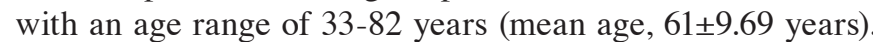
The standard for diagnosis in all cases was the combination of liquid-based cytology and pathology.

Cytology diagnosis. All materials were provided by LBP Medicine Science and Technology Co., Ltd (Guangzhou, China). All operations were completed at room temperature. Specimens were naturally precipitated for 10-15 min and then centrifuged at $1,610 \mathrm{x} \mathrm{g}$ for $5 \mathrm{~min}$ at room temperature. The supernatant was removed, and cell preservation solution provided by this company was applied to dilute the cells. The supernatant was removed if red blood cells could be observed in the lower layer, or else the reading result would be disrupted. The cells on the surface of the centrifuge tube were removed and the cell preservation solution was applied to dilute the cells. The final dilution concentration of cells was not specific provided it did not impact on their ability to be analysed. Slices (1.2 mm-thick) of BALF, effusion samples and FNA specimens were prepared, placed in 95\% alcohol and fixed for $>30 \mathrm{~min}$. All samples underwent Papanicolaou staining about $5 \mathrm{~min}$ at room temperature and were diagnosed by at least two pathologists from The First Affiliated Hospital of Chongqing Medical University (Chongqing, China), all of whom were blinded to the patients' data. If disagreements existed among the pathologists against results of specific samples, an additional pathologist then confirmed the findings. The results were classified as follows: No evidence of cancer cells; evidence of atypical cells; evidence of severe atypical cells; evidence of suspicious cancer cells; and presence of cancer cells (15). No evidence of cancer cells and evidence of atypical cells were regarded as negative, and the remaining categories were considered positive.

Automated DNA-ICM. The specimens underwent routine centrifugation at $800 \mathrm{x}$ g for $5 \mathrm{~min}$ in $35^{\circ} \mathrm{C}$ environment and the supernatant was discarded. Then, $100 \mu \mathrm{l}$ of cell dispersant purchased from Motic Electric (Xiamen, China) was added, and under the aforementioned centrifugation conditions, a cell precipitation solution was generated. After the addition of 2.5-3 times the volume of distilled water and agitation, the solution was resuspended. For the slides, 2-3 drops of cell suspension were dropped from $5 \mathrm{~cm}$ above the centre of the slide to complete specimen production. Then, all samples underwent Feulgen staining in $35^{\circ} \mathrm{C}$ environment using a $50 \%$ Feulgen stain, for 40-45 min. The nucleases on each slide were scanned using the SPICM-DNA automatic cell picture analysis system provided by Motic (Xiamen) Medical Diagnostic Systems Co., Ltd. (Xiamen, China) and the DNA of the nucleases was measured for standard comparisons with normal cells on the same slide. The DNA content of each cell nucleus was measured and DNA ploidy was recorded as a ' $c$ ' value. A DNA ploidy of $2 c$ indicates a normal diploid cell, and $4 c$ indicates a tetraploid cell. The DNA Index (DI) was utilized to present the contents of the cellular DNA, with a normal cellular DI=1 and a DI $>2.5$ indicating pathological cells (10). DI values of 1 and 2.5 reflected normal and suspicious pathological cells, respectively. The results of DNA-ICM were categorized into three classes: Negative results, cells without DNA aneuploidy; suspicious results, a small number of cells with DNA aneuploidy (DI $\geq 2.5$ for $1-2$ cells) and visible cellular abnormal hyperplasia $(\geq 10 \%)$; and highly suspicious tumor, visible DNA aneuploidy cells (DI $\geq 2.5$ for $\geq 3$ cells) with a visible aneuploidy cellular summit. No evidence of DNA aneuploidy cells was classified as a negative result, and evidence of suspicious or highly suspicious cells was classified as a positive result (Fig. 2).

Statistical analysis. The $\chi^{2}$-square test was used for the analysis of DNA-ICM with LBC results. The rank sum test was used to analyze the difference of the maximal DNA quantitation between squamous carcinoma and adenocarcinoma, and also between small cell lung cancer and non-small cell lung cancer. The correlations between tumor site, tumor type, clinical stage and the DNA quantitative maximum value, were analyzed using multivariate correlation analysis. Using a receiver operating characteristic (ROC) curve in order to evaluate the diagnostic accuracy of DNA-ICM with LBC. All statistical analyses were conducted using SPSS 20.0 software (IBM Corp., Armonk, NY, USA). All data is presented as mean \pm standard deviation. $\mathrm{P}<0.05$ was considered to indicate a statistically significant difference.

\section{Results}

Pathological diagnoses of all samples. The results of all pathological diagnosis (LBC and pathology) are illustrated in Table I. Out of the 465 cases, 176 cases were diagnosed as positive by LBC. A total of 289 cases exhibited no evidence of severe atypical cells or tumor cells and were diagnosed as negative by LBC. These results were consistent with the results of the pathological examinations showing 7 cases of squamous carcinomas, 11 cases of adenocarcinomas, 5 cases of small-cell carcinomas, 1 case of poorly differentiated carcinoma, 1 case of non-small cell lung cancer (NSCLC) and 1 unclassifiable tumor type case. The sensitivity of LBC was $87.13 \%$ (Table I).

Pathological diagnosis in BALF samples. Of the 385 BALF samples, 143 cases were diagnosed as positive by LBC. A total of 242 cases were diagnosed as negative by LBC. Based on the pathological biopsy results, 168 cases were diagnosed as positive. The sensitivity of LBC was $85.12 \%$ (Table I).

Pathological diagnosis in pleural effusion samples. Among the 70 pleural effusion samples, 26 cases were diagnosed as positive by $\mathrm{LBC}$ and 44 cases were diagnosed as negative. Based 


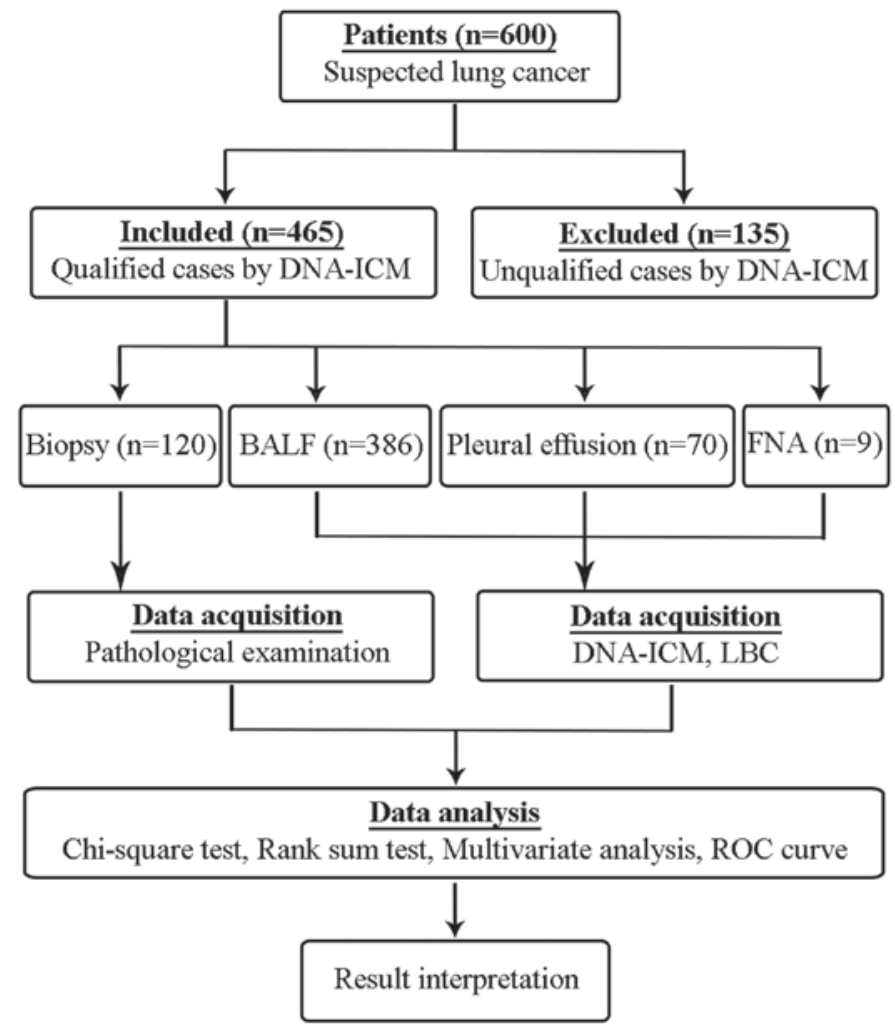

Figure 1. Flowchart of the research plan. BALF, bronchoalveolar lavage fluid; FNA, fine needle aspiration; LBC, liquid-based cytology; DNA-ICM, DNA image cytometry; ROC, receiver operating characteristic.

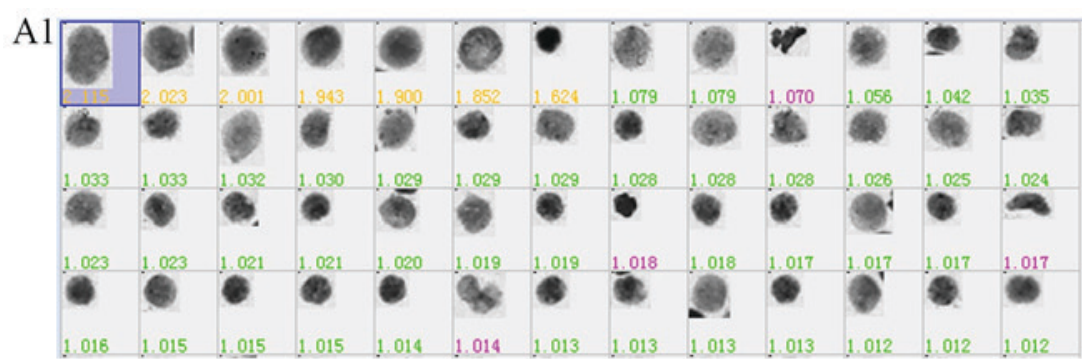

A2

DNA_index vs. area

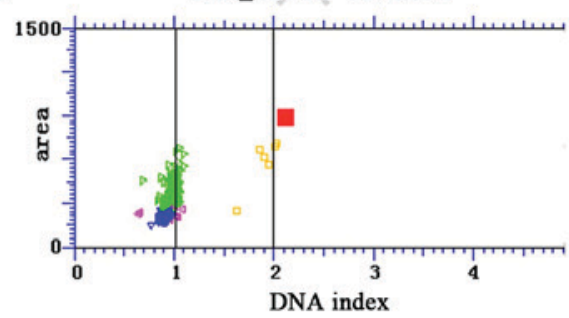

B1

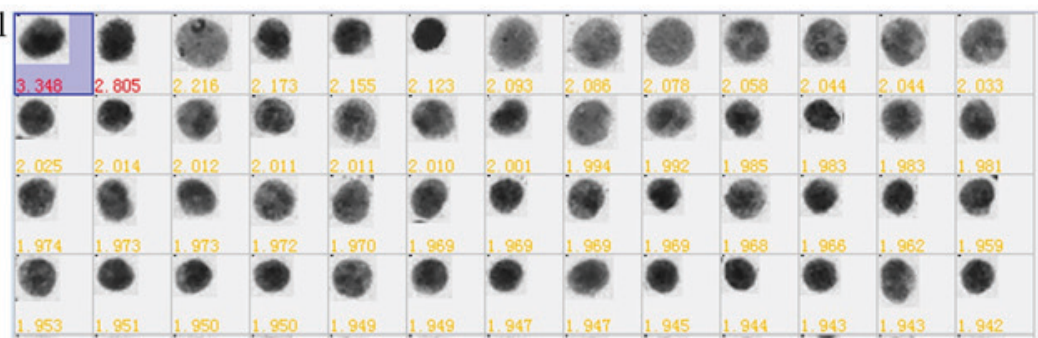

$\mathrm{Cl}$

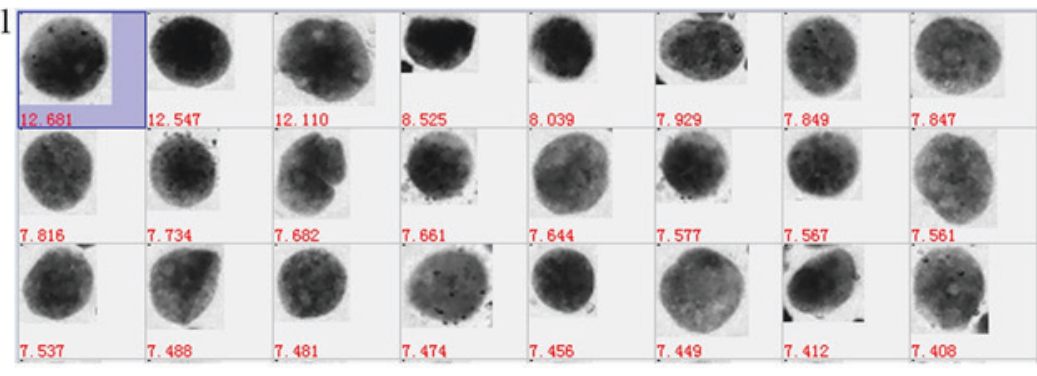

B2

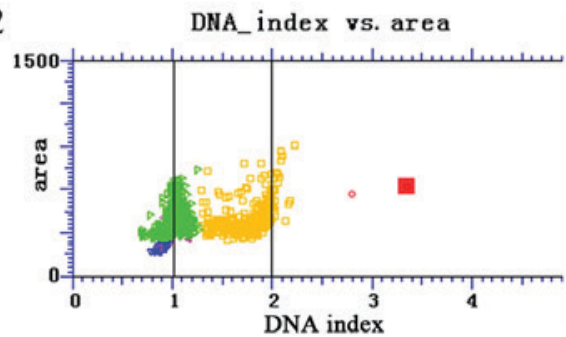

$\mathrm{C} 2$

DNA_index vs. area

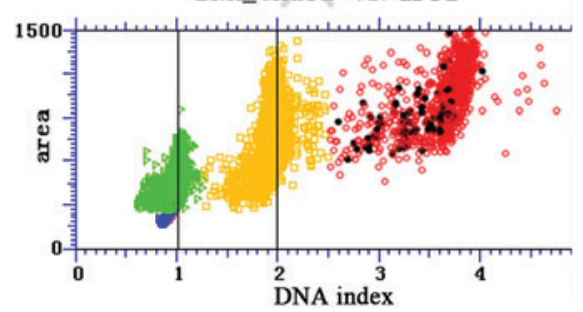

Figure 2. Difference between the results of the DNA-ICM. Representative (A1 and A2) negative; (B1 and B2) suspicious of tumor; and (C1 and C2) highly suspicious of tumor results. The leftimages illustrate the DNA index of every nucleus of different cells in the specimens. The right images illustrate the distribution of the cells in the specimens. Red represents pathological cells, orange represents hyperplastic cells, green represents normal cells, and blue and purple represent lymphocytes and neutrophils cells. The block diagram symbol in the images on the right represents the position of the cells and is representative of the selected cells. System scan cell under magnification, x20. 
Table I. Results of DNA-ICM, LBC and combined diagnosis in the three specimens of different tissue type.

\begin{tabular}{|c|c|c|c|c|c|c|c|c|}
\hline \multirow[b]{2}{*}{ Specimen type } & \multirow[b]{2}{*}{ Pathological diagnosis } & \multirow[b]{2}{*}{ Count } & \multicolumn{2}{|c|}{ DNA-ICM } & \multicolumn{2}{|c|}{ Cytology } & \multicolumn{2}{|c|}{ Combined diagnosi } \\
\hline & & & Positive & $\%$ & Positive & $\%$ & Positive & $\%$ \\
\hline \multicolumn{9}{|l|}{ BALF } \\
\hline & Squamous carcinoma & 64 & 46 & 71.87 & 57 & 89.06 & 60 & 93.75 \\
\hline & Adenocarcinoma & 56 & 35 & 62.5 & 45 & 80.36 & 49 & 87.5 \\
\hline & Small cell carcinoma & 32 & 23 & 71.88 & 28 & 87.5 & 30 & 93.75 \\
\hline & Non-small cell lung cancer & 9 & 6 & 66.67 & 8 & 99.89 & 8 & 99.89 \\
\hline & Poor differentiated carcinoma & 6 & 6 & 100 & 5 & 83.33 & 6 & 100 \\
\hline & Unclassifiable & 1 & 0 & 0 & 0 & 0 & 0 & 0 \\
\hline & Total & 168 & 116 & 69.04 & 143 & 85.12 & 153 & 91.07 \\
\hline \multicolumn{9}{|l|}{ Pleural effusion } \\
\hline & Squamous carcinoma & 1 & 1 & 100 & 1 & 100 & 1 & 100 \\
\hline & Adenocarcinoma & 23 & 21 & 91.3 & 23 & 100 & 23 & 100 \\
\hline & Small cell carcinoma & 2 & 0 & 0 & 1 & 50 & 1 & 50 \\
\hline & Poor differentiated carcinoma & 1 & 1 & 100 & 1 & 100 & 1 & 100 \\
\hline & Total & 27 & 24 & 88.89 & 26 & 96.3 & 26 & 96.3 \\
\hline \multicolumn{9}{|l|}{ FNA } \\
\hline & Squamous carcinoma & 4 & 3 & 75 & 4 & 100 & 4 & 100 \\
\hline & Adenocarcinoma & 2 & 1 & 50 & 2 & 100 & 2 & 100 \\
\hline & Small cell carcinoma & 1 & 1 & 100 & 1 & 100 & 1 & 100 \\
\hline & Total & 7 & 5 & 71.42 & 7 & 100 & 7 & 100 \\
\hline \multicolumn{9}{|l|}{ Overall } \\
\hline & Squamous carcinoma & 69 & 50 & 72.46 & 62 & 89.86 & 65 & 94.2 \\
\hline & Adenocarcinoma & 81 & 57 & 70.37 & 70 & 86.42 & 74 & 91.36 \\
\hline \multirow[t]{3}{*}{ NSCLC } & Poor differentiated carcinoma & 7 & 7 & 100 & 6 & 85.71 & 7 & 100 \\
\hline & Unclassifiable & 1 & 0 & 0 & 0 & 0 & 0 & 0 \\
\hline & Non-small cell lung cancer & 9 & 6 & 66.67 & 8 & 88.89 & 8 & 88.89 \\
\hline \multirow[t]{2}{*}{ SCLC } & Small cell carcinoma & 35 & 25 & 71.42 & 30 & 85.71 & 32 & 91.43 \\
\hline & Total & 202 & 145 & 71.78 & 176 & 87.13 & 186 & 92.08 \\
\hline
\end{tabular}

BALF, bronchoalveolar lavage fluid; DNA-ICM, DNA image cytometry-FNA, fine-needle aspiration.

on the pathological biopsy results, 27 cases were diagnosed as positive. The sensitivity of LBC was $96.30 \%$ (Table I).

Pathological diagnosis in FNA samples. The results of LBC and pathological examinations were the same, with 2 cases diagnosed as negative by LBC and 7 cases diagnosed as positive. The sensitivity of LBC was $100 \%$ (Table I).

Results of DNA-ICM. The results of DNA-ICM for all types of specimens are shown in Table II. There were 194 cases diagnosed as positive by DNA-ICM and 271 cases diagnosed as negative. There were 49 cases diagnosed as positive by DNA-ICM and negative by LBC, but no pathology results were available. There were 57 cases of false-negative. In 10 cases of positive samples diagnosed by pathological biopsy, the LBC results were negative while the DNA-ICM results were positive.

Table II shows the positive diagnostic rates of DNA-ICM, LBC and the combined approach in specimens from three different tissue types. The sensitivities of LBC and DNA-ICM for BALF samples were 85.12 and $69.04 \%$, respectively, and were significantly different $\left(\mathrm{X}^{2}=12.282 ; \mathrm{P}<0.05\right)$. The sensitivities of LBC and the combined approach for BALF samples were 85.12 and $91.04 \%$, respectively, and were not significantly different $\left(\mathrm{X}^{2}=2.838 ; \mathrm{P}>0.05\right)$. Furthermore, the sensitivities of DNA-ICM for BALF samples in detecting lung squamous carcinoma and lung adenocarcinoma were 71.87 and $62.5 \%$, with no statistically significant difference.

In the positive pleural effusion specimens, the sensitivities of LBC and DNA-ICM were 96.3 and $88.89 \%$, respectively, and were not significantly different $\left(\mathrm{X}^{2}=1.080 ; \mathrm{P}>0.05\right)$. The sensitivities of LBC and the combined approach were the same, with sensitivities for lung squamous carcinoma, and lung adenocarcinoma of 100 and $91.3 \%$, respectively, with no statistically significant difference $(\mathrm{X}=0.095 ; \mathrm{P}>0.05)$. The sensitivities of the DNA-ICM method for BALF and pleural effusion samples were 69.04 and $88.89 \%$, respectively, and were not significantly different $\left(\mathrm{X}^{2}=4.522 ; \mathrm{P}>0.05\right)$. The number of lung biopsy specimens was too small for a separate analysis. 
Table II. Results of DNA-ICM and pathological diagnosis for all type of specimens.

\begin{tabular}{|c|c|c|c|c|c|}
\hline \multirow[b]{2}{*}{ Specimen type } & \multirow[b]{2}{*}{ DNA-ICM } & \multicolumn{2}{|c|}{ Pathology } & \multirow[b]{2}{*}{ Sensitivity (\%) } & \multirow[b]{2}{*}{ Specificity (\%) } \\
\hline & & Positive & Negative & & \\
\hline \multicolumn{6}{|l|}{ Overall } \\
\hline & Positive & 145 & 49 & 71.78 & 81.37 \\
\hline & Negative & 57 & 214 & & \\
\hline \multicolumn{6}{|l|}{ BALF } \\
\hline & Positive & 116 & 43 & 69.05 & 80.28 \\
\hline & Negative & 52 & 175 & & \\
\hline \multicolumn{6}{|l|}{ Pleural effusion } \\
\hline & Positive & 24 & 6 & 88.89 & 86.05 \\
\hline & Negative & 3 & 37 & & \\
\hline \multicolumn{6}{|l|}{ FNA } \\
\hline & Positive & 5 & 0 & 71.43 & 100 \\
\hline & Negative & 2 & 2 & & \\
\hline
\end{tabular}

Table III. The result of tumor staging, tumor location, and the type.

\section{NSCLC}

\begin{tabular}{|c|c|c|c|c|}
\hline \multirow[b]{2}{*}{ Variable } & & \multirow[b]{2}{*}{ SCLC } \\
\hline & Squamous carcinoma & Adenocarcinoma & Other & \\
\hline \multicolumn{5}{|l|}{ Stage } \\
\hline I & 1 & 7 & 0 & \\
\hline II & 2 & 0 & 2 & \\
\hline III & 17 & 3 & 3 & \\
\hline IV & 22 & 36 & 4 & \\
\hline Extensive stage & & & & 19 \\
\hline Limited period & & & & 7 \\
\hline \multicolumn{5}{|l|}{ Location } \\
\hline Central & 25 & 18 & 5 & 20 \\
\hline Peripheral & 16 & 28 & 5 & 6 \\
\hline
\end{tabular}

NSCLC, non small cell lung cancer; SCLC, small cell lung cancer.

The total results of DNA-ICM, LBC and the combined approach for all tumor types in all specimens are shown in Table I. In positive specimens, the sensitivities of LBC and DNA-ICM were 87.13 and $71.78 \%$, respectively, with a statistically significant difference $\left(\mathrm{X}^{2}=14.572 ; \mathrm{P}<0.05\right)$. No statistically significant difference was observed in the positive diagnostic rates between $\mathrm{LBC}$ and the combined approach $\left(\mathrm{X}^{2}=2.657 ; \mathrm{P}>0.05\right)$. The differences in the positive diagnostic rates of DNA-ICM for squamous carcinoma and adenocarcinoma were not statistically significant $\left(\mathrm{X}^{2}=0.80\right.$; $\mathrm{P}>0.05)$.

Overall, 202 cases of lung cancer were identified by cytology and pathology. In these cases, DNA-ICM has certain missed rates, whereby 19/69 cases of squamous cell carcinoma and 24/81 cases of adenocarcinoma were missed. The positive diagnosis rate of DNA-ICM in squamous cell carcinoma and adenocarcinoma cell carcinoma was not statistically significant $(\mathrm{P}>0.05)$. In addition, $10 / 35$ cases of small cell lung cancer (SCLC) were missed, 46/167 cases of non-small cell lung cancer (NSCLC, all types of lung cancer outside of SCLC) were missed. Between the positive diagnosis rate of SCLC and NSCLC, there was no significant difference $(\mathrm{P}>0.05)$ (Table I).

Table III showed the results of tumor staging, tumor location, and the type. Association between the maximum value of DNA (DNAmax) and tumor staging, tumor location, and tumor type. Of the 202 lung cancer cases, due to economic factors, clear tumor staging was achieved in 123 cases by relevant 
A

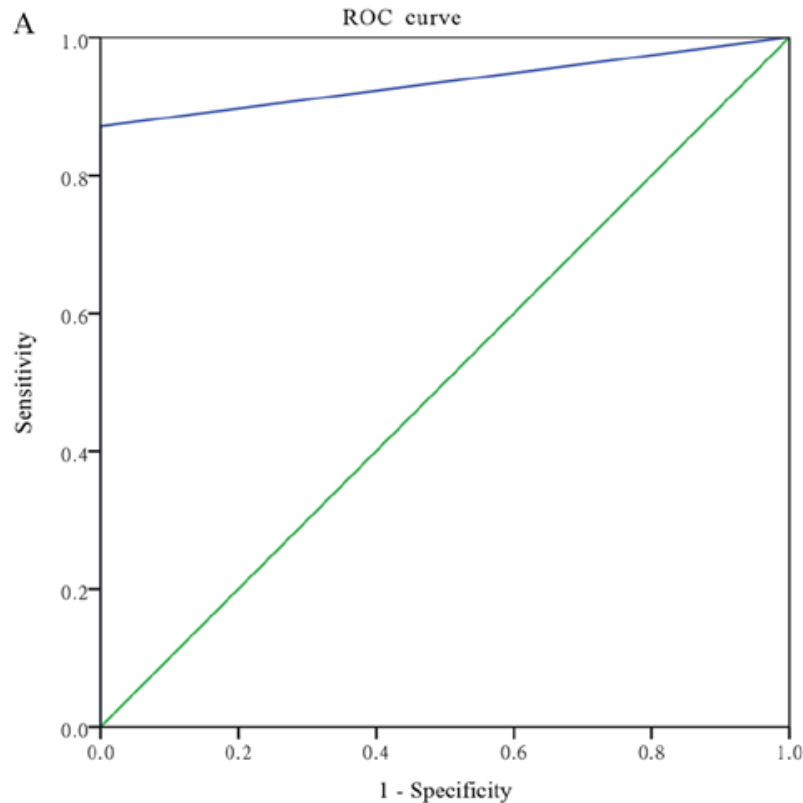

B

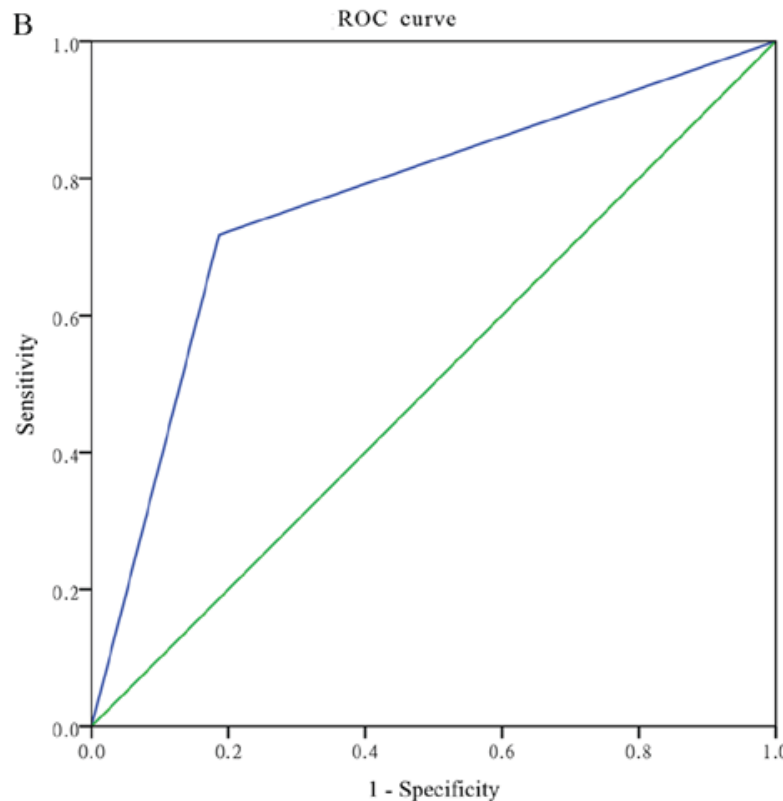

Figure 3. ROC curves of the LBC and DNA-ICM. ROC curves for (A) liquid-based cytology and (B) DNA image cytometry. ROC, receiver operating characteristic; LBC, liquid-based cytology; DNA-ICM, DNA image cytometry

examinations, including 97 cases of NSCLC of stages I to IV (I, 8 cases; II, 4 cases; III, 23 cases; IV, 62 cases), with the tumor site categorized as central (48 cases) or peripheral (49 cases). There were 26 cases of small cell lung cancer (SCLC), which were divided into extensive stage (19 cases) and limited period ( 7 cases), with the tumor site categorized as central (20 cases) or peripheral (6 cases). The correlation coefficient between DNAmax and tumor stage was $0.305(\mathrm{P}=0.002)$, which was positively correlated with NSCLC, but there was no significant correlation between DNAmax and tumor type or tumor location. In SCLC cases, DNAmax, tumor staging and tumor site were not significantly correlated.

The difference in DNAmax between squamous cell carcinoma and adenocarcinoma was analysed by the rank sum test, and was not statistically significant $(\mathrm{P}=0.054)$. DNAmax values in SCLC and NSCLC were analysed, and the difference was not statistically significant $(\mathrm{P}=0.359)$.

ROC curves for LBC and DNA-ICM. A total of 202 patients diagnosed with lung cancer, including 26 LBC negative cases and 57 DNA-ICM negative cases. Among the 263 cases of pathologically undiagnosed lung cancers, there were 49 abnormal cases suggested by DNA-ICM. Then the ROC curves were drew according to the results of the test. The areas under the ROC curves of LBC and DNA-ICM were 0.936 and 0.766 , respectively (Fig. 3), with significant difference $(\mathrm{P}<0.05)$. It can be indicated that $\mathrm{LBC}$ is the optimal method to diagnose lung cancer, seconded by DNA-ICM method.

\section{Discussion}

DNA is the foundation of cell growth, differentiation and reproduction. It exists in a diploid state in the normal body and becomes tetraploid when mitosis begins. However, when a cell undergoes carcinogenesis, genes go through various changes, including mutation, recombination, deficiency, fusion and amplification, resulting in increased cellular DNA content. Therefore, determining the DNA content of target cells relative to normal cells as standard controls may aid in diagnosing an early-stage tumor $(5,6)$. DNA quantitative analysis is a method that maybe mastered in a short time period, with the characteristics of objectivity, quickness, independence and effectiveness (7-10). DNA-ICM has been applied in research on cervical, oesophageal and oral mucosal cancer, and has been gradually implemented into the clinic, but it has not been used for diagnosing lung carcinoma as of yet (11-14).

Histopathology has long been regarded as the gold standard for detecting cancer (3). LBC is also a well-known diagnostic standard that has been used for the clinical diagnosis of lung carcinoma (16). DNA-ICM and LBC require a wait time of $<6 \mathrm{~h}(17)$ and are priced similarly at $\sim 500 \mathrm{RMB}$. However, the DNA detection method is relatively simple and the training of specialized staff requires a few weeks. LBC diagnoses rely on the judgement of pathologists who require several years of specialized training (9). However, DNA-ICM can only judge the abnormal cells, but it cannot identify the types of tumor cell, including squamous carcinoma, adenocarcinoma and SCLC.

In the present study, LBC and DNA-LBC results were available for all cases, and histopathology results were available for 120 cases. Therefore, the present study aimed to analyse the diagnostic value of DNA-ICM in the diagnosis of lung carcinoma compared with the combined approach of LBC and histopathology.

Certain malignant tumors, including lung cancer, exhibit diploid DNA, whereas certain benign cases present heteroploid cells (18-21). Rijken et al (19) reported that $25 \%$ of malignant tumors are diploid, including in lung, oral, breast and bladder cancer (19,21-25). In the current study, 202 confirmed lung cancer cases were identified. Correlation analysis demonstrated that DNAmax and tumor staging were positively correlated in NSCLC cases, possibly because the tumor was 
more aggressive. This result is similar to the findings of a study by Kasprzyk et al (26).

The DNA analysis results revealed that the following cases were not heteroploid: 52 cases with BALF samples; 3 cases with pleural effusion samples; and 2 cases with FNA samples. Evident heteroploid cells were observed in 49 cases, but no pathological evidence of lung cancer was observed.

In the 57 false-negative cases from the DNA-ICM results, one case evaluated using a pleural effusion sample was diagnosed as small-cell carcinoma; however, this patient is currently being treated with chemotherapy. Chemotherapy may affect tumor ploidy (27), which can affect DNA analysis results. Various studies have also reported that no heteroploidy is evident in certain DNA samples of malignant tumors with different ratios of false-negative cases (19,21-23). There are also reports indicating that in certain cells, due to similar appearances, DNA analysis results may demonstrate heteroploidy and diploidy because of their heterogenicity or because diploids are able to gradually become heteroploids during tumor development $(22,28,29)$. However, the study of Bisht et al (23) reported that certain minor changes and chromosomal abnormalities cannot be detected by DNA analysis. When the number of diploid cells predominates over that of heteroploid cells, DNA analysis may still not detect abnormal cells $(21,30)$. In addition, DNA quantitative analysis does not reveal heteroploid cells if the tumor chromosomes maintain a balance between loss and replication $(18,31)$. Therefore, so-called false-negative results may not truly be false-negative under the circumstance that tumor characteristics cannot be detected by DNA-ICM. In the present study, the majority of the false-negative results came from BALF samples as there is no specific standard for BALF retrieval, and samples are not typically collected by the same person, leading to differences in surgery technique. All these factors may influence sample quality. In addition, the effects of sampling errors and poor sample handling could not be excluded (14), which may lead to real false-negative results.

A total of 49 cases, included routine follow-up visit data. DNA analysis revealed abnormalities, but none of these cases were diagnosed as lung cancer. Ten patients showed a high possibility of lung cancer based on their medical histories, tumor biomarker results and imaging results. However, specific pathological evidence for their diagnoses was not obtained, as the patients refused to undergo tissue biopsy. Furthermore, the remaining 39 cases, 3 cases of pulmonary tuberculosis, 19 cases of pulmonary infection, 6 cases of pulmonary benign changes, and 11 undiagnosed cases, demonstrated heteroploidy even though they were not diagnosed as lung cancer, possibly because DNA-ICM is able to diagnose malignant tumors 1-15 months before histology can $(32,33)$. Furthermore, a previous study reported the possibility of false-positive results caused by hyperplasia when cells are affected by bacteria, viruses, fungus or certain drugs (10). Heteroploidy may also appear when cells are damaged during the process of obtaining samples. The aforementioned factors may account for the appearance of false-positive results to a certain extent. The present cohort of patients needs to be followed to determine whether lung cancer or another disease maybe diagnosed.

The present results suggest that the diagnostic value of DNA-ICM may not be superior to LBC, according to the positive diagnostic rates and ROC curves. In pleural effusion samples, the results of DNA-ICM were the same as those of pathology, but the positive diagnostic rate of DNA-ICM was significantly lower compared with that of LBC in BALF samples, potentially due to the aforementioned reasons. In 10 BALF samples, cancer cells were not detected by LBC, while DNA-ICM revealed heteroploid cells in tissue biopsies, thus confirming the presence of lung cancer. This may be explained by the fact that LBC results are interpreted according to a physicians' visual evaluation, which may lead to subjective outcomes and missed diagnoses, but DNA-ICM is able to identify precancerous lesions that are overlooked in visual inspection. Therefore, LBC combined with DNA-ICM improves the positive diagnostic rate, even though there is no significant difference when compared with LBC alone. In addition, 16 cases were identified as negative by LBC and DNA-ICM, but the tissue biopsies revealed lung cancer. Therefore, tissue biopsies are still necessary to diagnose lung cancer and to avoid missed diagnoses, even when LBC and DNA-ICM are combined.

DNA-ICM has been widely used in the diagnosis of the cervical cancer, with a threshold of $>5 \mathrm{c}$ for DNA content and DI $\geq 3$ for cell amount (11). Furthermore, Bradley et al (13) reported that the amount of DNA is $>4.6 \mathrm{C}$ and the cell amount is $\geq 3$ in oral cancer, which is regarded as the positive threshold. Tao et al (34) reported that a DNA content of $>2.5 \mathrm{C}$ and cell amount of $\geq 4$ had the highest diagnostic value in BALF samples (34). Therefore, different types of malignant tumors have various thresholds in DNA-ICM. However, the diagnostic threshold for lung cancer has not yet been standardized. In the current study, a diagnostic threshold of $>5 \mathrm{C}$ for DNA content and $\geq 3$ for cell amount was chosen, and positive results were determined when the DNA-ICM results suggested the presence of cancer. The appearance of false-positive and false-negative results in the present study may be associated with the difference in the diagnostic threshold. In the present study, cases with false-negative results accounted for majority of the BALF samples, possibly because different types of samples require different diagnostic thresholds; however, more samples are required to determine the reason behind this finding.

Tao et al (34) reported that the positive diagnostic rate for SCLC is significantly higher compared with that for pulmonary adenocarcinoma in BALF samples. Nevertheless, in the results of the present study, no statistically significant difference was identified between these two types of cancer in BALF and pleural effusion samples, which may be due to the diagnostic threshold chosen, and sampling errors. Therefore, more samples must be collected to determine the optimal diagnostic threshold.

Certain researchers suggest that due to tumor heterogeneity, it is necessary to perform DNA-ICM on five independent samples for every case to identify true diploid cells and decrease the likelihood of false-negative results $(22,29)$. However, this would greatly increase examination costs. Additionally, DNA-ICM may only determine differences in DNA amounts rather than the exact pathological types of cancer. Therefore, further examinations with cytology or tissue biopsy are still required to define the cancer type. The clinical application of DNA-ICM is indeed restricted in this 
way. However, DNA-ICM may serve as a valuable method when utilized appropriately.

Automatic DNA-ICM has medium diagnostic value in lung cancer. The positive diagnostic rate in lung cancer maybe improved to a certain extent when DNA-ICM is combined with LBC, demonstrating a possible supplementary role. DNAmax was positively correlated with tumor stage in NSCLC, but DNA-ICM is not able to identify the type of tumor, and may generate false-positive and false-negative results. Therefore, DNA-ICM alone cannot be recommended for lung cancer screening. The threshold value of DNA-ICM for lung specimens is not yet clear and requires further study. When the results of LBC and DNA-ICM are conflicting, the patient should be thoroughly evaluated.

\section{Acknowledgements}

The authors would like to thank the staff of The First Affiliated Hospital of Chongqing Medical University and Motic Medical Diagnostic Systems Co., Ltd. for specimen collection and analysis.

\section{Funding}

Chongqing Science and Technology application development project (Chongqing, China; grant no. cstc2014yykfA110026).

\section{Availability of data and materials}

The datasets used and analysed during the current study are available from the corresponding author on reasonable request.

\section{Authors' contributions}

TJ conducted the experiments. AS participated in collecting data and drafted the manuscript. WM participated in collecting data and helped revise the manuscript. WX and LX contributed to statistical analysis and manuscript writing. All authors read and approved the final manuscript.

\section{Ethics approval and consent to participate}

The present study was approved by the Ethics Committee of the First Affiliated Hospital of Chongqing Medical University (Chongqing, China). Written informed consent was obtained from all patients.

\section{Consent for publication}

Patient, parent or next of kin provided written informed consent for the publication of any associated data and accompanying images.

\section{Competing interests}

The authors declare that they have no conflicts of interests.

\section{References}

1. Jemal A, Bray F, Center MM, Ferlay J, Ward E and Forman D: Global cancer statistics. CA Cancer J Clin 61: 69-90, 2011.
2. Ferlay J, Shin HR, Bray F, Forman D, Mathers C and Parkin DM: Estimates of worldwide burden of cancer in 2008: GLOBOCAN 2008. Int J Cancer 127: 2893-2917, 2010.

3. Travis WD, Rekhtman N, Riley GJ, Geisinger KR, Asamura H, Brambilla E, Garg K, Hirsch FR, Noguchi M, Powell CA, et al: Pathologic diagnosis of advanced lung cancer based on small biopsies and cytology: A paradigm shift. J Thorac Oncol 5: 411-414, 2010.

4. Luo Q, Shao S, Chen Y, et al: Value of bronchial brushing liquid-based cytology in diagnosis of lung cancer. J Mod Oncol 23: 2129-2132, 2015.

5. Li G, Guillaud M, Follen M and MacAulay C: Double staining cytologic samples with quantitative Feulgen-thionin and anti-Ki-67 immunocytochemistry as a method of distinguishing cells with abnormal DNA content from normal cycling cells. Anal Quant Cytopathol Histpathol 34: 273-284, 2012.

6. D'Urso V, Collodoro A, Mattioli E, Giordano A and Bagella L: Cytometry and DNA ploidy: Clinical uses and molecular perspective in gastric and lung cancer. J Cell Physiol 222: 532-539, 2010.

7. Tong H, Shen R, Wang Z, Kan Y, Wang Y, Li F, Wang F, Yang J and Guo X; Mass Cervical Cancer Screening Regimen Group: DNA ploidy cytometry testing for cervical cancer screening in China (DNACIC Trial): A prospective randomized, controlled trial. Clin Cancer Res 15: 6438-6445, 2009.

8. Nghiem VT, Davies KR, Beck JR, Follen M, MacAulay C, Guillaud $\mathrm{M}$ and Cantor SB: Economic evaluation of DNA ploidy analysis vs liquid-based cytology for cervical screening. Br J Cancer 112: 1951-1957, 2015.

9. Wang M, Hao C, Ma Q, Song G, Ma S, Zhao D, Zhao L, Li X and Wei W: DNA image cytometry test for primary screening of esophageal cancer: A population-based multi-center study in high-risk areas in China. Chin J Cancer Res 28: 404-412, 2016.

10. Garner D: Clinical application of DNA ploidy to cervical cancer screening: A review. World J Clin Oncol 5: 931-965, 2014.

11. Guillaud M, Benedet JL, Cantor SB, Staerkel G, Follen M and Macaulay C: DNA ploidy compared with human papilloma virus testing (Hybrid Capture II) and conventional cervical cytology as a primary screening test for cervical high-grade lesions and cancer in 1555 patients with biopsy confirmation. Cancer 107: 309-318, 2006.

12. Zhao L, Wei WQ, Zhao DL, Hao CQ, Lin DM, Pan QJ, Li XQ, Lei FH, Wang JW, Wang GQ, et al: Population-based study of DNA image cytometry as screening method for esophageal cancer. World J Gastroenterol 18: 375-382, 2012.

13. Bradley G, Odell EW, Raphael S, Ho J, Le LW, Benchimol S and Kamel-Reid S: Abnormal DNA content in oral epithelial dysplasia is associated with increased risk of progression to carcinoma. Br J Cancer 103: 1432-1442, 2010.

14. Kämmerer PW, Koch FP, Santoro M, Babaryka G, Biesterfeld S, Brieger J and Kunkel M: Prospective, blinded comparison of cytology and DNA-image cytometry of brush biopsies for early detection of oral malignancy. Oral Oncol 49: 420-426, 2013.

15. Guo R, Yang J, Baoshan SU, et al: Automatic DNA image cytometry can improve the positive detectable rate of cancer by fiberobronchoscopy. J Shanxi Med Univ, 2016.

16. Michael CW and Bedrossian CC: The implementation of liquid-based cytology for lung and pleural-based diseases. Acta Cytol 58: 563-573, 2014.

17. Baak JPA and Janssen E: DNA ploidy analysis in histopathology. Morphometry and DNA cytometry reproducibility conditions and clinical applications. Histopathology 44: 603-614, 2004.

18. Esteban JM and Sheibani K: DNA ploidy analysis of pleural mesotheliomas: Its usefulness for their distinction from lung adenocarcinomas. Mod Pathol 5: 626-630, 1992.

19. Rijken A, Dekker A, Taylor S, Hoffman P, Blank M and Krause JR: Diagnostic value of DNA analysis in effusions by flow cytometry and image analysis. A prospective study on 102 patients as compared with cytologic examination. Am J Clin Pathol 95: 6-12, 1991.

20. Unger KM, Raber M, Bedrossian CW, Stein DA and Barlogie B: Analysis of pleural effusions using automated flow cytometry. Cancer 52: 873-877, 1983.

21. Lazcano O, Chen LM, Tsai C, Li CY, Katzmann JA, Sebo TJ, Kimlinger TK and Baker J: Image analysis and flow cytometric DNA studies of benign and malignant body cavity fluids: Reappraisal of the role of current methods in the differential diagnosis of reactive versus malignant conditions. Mod Pathol 13: 788-796, 2000 
22. Diwakar N, Sperandio M, Sherriff M, Brown A and Odell EW: Heterogeneity, histological features and DNA ploidy in oral carcinoma by image-based analysis. Oral Oncol 41: 416-422, 2005.

23. Bisht B, Handa U, Mohan $\mathrm{H}$ and Lehl SS: Complementary value of DNA flow cytometry and image morphometry in detection of malignant cells in effusion fluids. Malays J Pathol 36: 83-90, 2014.

24. Schneller J, Eppich E, Greenebaum E, Elequin F, Sherman A, Wersto R and Koss LG: Flow cytometry and Feulgen cytophotometry in evaluation of effusions. Cancer 59: 1307-1313, 1987.

25. Kaur M, Handa U, Mohan H and Dass A: Evaluation of brush cytology and DNA image cytometry for the detection of cancer of the oral cavity. Diagn Cytopathol 44: 201-205, 2016.

26. Kasprzyk M, Dyszkiewicz W, Piwkowski C, Gasiorowski L and Kaczmarek E: Prognostic value of DNA ploidy: 5-year follow-up of patients with resectable squamous cell carcinoma (SCC) of the lung. Lung Cancer 51: 201-206, 2006.

27. Kubota K, Kajiura N, Konishi T, Teruya M, Tsushima H, Nakao K, Arizono S, Oka T and Makuuchi M: Effects of preoperative chemotherapy on DNA ploidy patterns, cell cycle, and histological findings in gastric and colonic cancer patients. J Surg Oncol 57: 222-229, 1994

28. Baba H, Korenaga D, Kakeji Y, Haraguchi M, Okamura T and Maehara Y: DNA ploidy and its clinical implications in gastric cancer. Surgery 131 (1 Suppl): S63-S70, 2002.
29. Furuya T, Uchiyama T, Murakami T, Adachi A, Kawauchi S, Oga A, Hirano T and Sasaki K: Relationship between chromosomal instability and intratumoral regional DNA ploidy heterogeneity in primary gastric cancers. Clin Cancer Res 6: 2815-2820, 2000

30. Ma JM, Zhou TJ, Wang R, Shan J, Wu YN, Song XL, Gu N and Fan Y: Brush biopsy with DNA-image cytometry: A useful and noninvasive method for monitoring malignant transformation of potentially malignant oral disorders. Eur Arch Otorhinolaryngol 271: 3291-3295, 2014.

31. Fan Y and Li L: The application of cytometric DNA analysis in the diagnosis of malignant pleural effusions. Oncology Progress, 2005.

32. Remmerbach TW, Weidenbach H, Hemprich A and Böcking A: Earliest detection of oral cancer using non-invasive brush biopsy including DNA-image-cytometry: Report on four cases. Anal Cell Pathol 25: 159-166, 2003.

33. Maraki D, Becker J and Boecking A: Cytologic and DNA-cytometric very early diagnosis of oral cancer. J Oral Pathol Med 33: 398-404, 2004.

34. Tao W, Li J and Cheng LL: Application of DNA-image cytometry in the diagnosis of lung cancer. Zhonghua Zhong Liu Za Zhi 38: 113-117, 2016 (In Chinese).

(i) (9) This work is licensed under a Creative Commons (c) ${ }_{\mathrm{EY}} \mathrm{NO}$ No Attribution-NonCommercial-NoDerivatives 4.0 International (CC BY-NC-ND 4.0) License. 